\title{
DISTANCE FUNCTIONS AND ALMOST GLOBAL SOLUTIONS OF EIKONAL EQUATIONS
}

\author{
LUIS A. CAFFARELLI \\ MICHAEL G. CRANDALL
}

\begin{abstract}
Consider a function $u$ defined on $\mathcal{R}^{n}$, except, perhaps, on a closed set of potential singularities $\mathcal{S}$. Suppose that $u$ solves the eikonal equation $\|D u\|=1$ in the pointwise sense on $\mathcal{R}^{n} \backslash \mathcal{S}$, where $D u$ denotes the gradient of $u$ and $\|\cdot\|$ is a norm on $\mathcal{R}^{n}$ with the dual norm $\|\cdot\|_{*}$. For a class of norms which includes the standard $p$-norms on $\mathcal{R}^{n}, 1<p<\infty$, we show that if $\mathcal{S}$ has Hausdorff 1-measure zero and $n \geq 2$, then $u$ is either affine or a "cone function," that is, a function of the form $u(x)=a \pm\|x-z\|_{*}$.
\end{abstract}

\section{INTRODUCTION}

The function

$$
u(x)=\langle x, p\rangle \quad \text { for } \quad x \in \mathcal{R}^{n},
$$

where $p \in \mathcal{R}^{n}$ and $\langle x, p\rangle$ is the Euclidean inner-product of $x$ and $p$, satisfies $D u=p$ and $|D u|=|p|$ on $\mathcal{R}^{n}$. Here $D u$ is the gradient of $u$ and $|\cdot|$ is the Euclidean norm. If $|p|=1$, then $u \in C^{1}\left(\mathcal{R}^{n}\right)$ and $|D u|=1$ everywhere. If $z \in \mathcal{R}^{n}$, the functions

$$
u(x)=a \pm|x-z|,
$$

where $a \in \mathcal{R}$, satisfy $u \in C^{1}\left(\mathcal{R}^{n} \backslash\{z\}\right)$. Moreover,

$$
D u(x)= \pm \frac{x-z}{|x-z|} \Longrightarrow|D u(x)|=1 \quad \text { for } \quad x \neq z .
$$

We call the special solutions (1.1) of $|D u|=1$ cone functions.

The distance to the line segment joining $(-1,0, \ldots, 0)$ and $(1,0, \ldots, 0)$,

$$
u(x)=d(x,\{(s, 0, \ldots, 0):-1 \leq s \leq 1\}),
$$

is given by

$$
u\left(x_{1}, x_{2}, \ldots, x_{n}\right)=\left\{\begin{array}{l}
\left(x_{2}^{2}+\ldots+x_{n}^{2}\right)^{1 / 2} \text { if }\left|x_{1}\right| \leq 1 \\
\left(\left(x_{1}-1\right)^{2}+x_{2}^{2}+\ldots+x_{n}^{2}\right)^{1 / 2} \text { if } x_{1} \geq 1, \\
\left(\left(x_{1}+1\right)^{2}+x_{2}^{2}+\ldots+x_{n}^{2}\right)^{1 / 2} \text { if } x_{1} \leq-1 .
\end{array}\right.
$$

In this case, $u$ is $C^{1}$ off the segment and satisfies $|D u|=1$ there; moreover, $u$ is not differentiable at any point of the segment.

Acknowledgements: The first author was partially supported by NSF Grant DMS-0654267 and the second by DMS-0400674. The second author thanks the Department of Mathematics, University of Texas at Austin, for its hospitality on several visits during this work. 
The linear function has no singularities, the cone functions have one singularity, and the distance to the line segment has a one dimensional set of singularities. One may ask whether it is possible for the set of singularities to be larger than a singleton but "genuinely" smaller than a line segment. If $n=1$, the distance function to the integers has countably many singularities. However, if $n \geq 2$, the answer is no, with the appropriate notion of "smaller." More precisely, we will prove that if $n \geq 2$ and $\mathcal{S}$ is a closed subset of $\mathcal{R}^{n}$ of Hausdorff 1-measure 0 (our notion of "almost global"), and $u$ is differentiable and satisfies $|D u|=1$ on $\mathcal{R}^{n} \backslash \mathcal{S}$, then $u$ is either affine or has a single singularity, in which case $u(x)$ is a cone function. An interesting sidelight is that if $\mathcal{S}=\emptyset$, that is, $u$ is a global solution, then $u$ is affine. This last remark is true quite generally, see Remark 2.3 of Section 2.

In fact, we will prove a full generalization of this result for equations of the form

$$
\|D u\|=1
$$

where $\|\cdot\|$ is a strictly convex norm which is differentiable off the origin, provided that the norm dual to $\|\cdot\|$, $\|\cdot\|_{*}$, as given by

$$
\|x\|_{*}:=\max _{\|y\|=1}\langle x, y\rangle
$$

is geometrically convex and balanced. The terms "geometrically convex" and "balanced" were introduced in Caffarelli and Crandall [2]; the definitions are recalled in Section 4. For the moment, let us just say that this generality allows $\|\cdot\|$ to be any of the $p$ norms $\|\cdot\|_{p}, 1<p<\infty$, where

$$
\|x\|_{p}:=\left(\left|x_{1}\right|^{p}+\cdots+\left|x_{n}\right|^{p}\right)^{1 / p},
$$

and that the extension of the Euclidean results to cover all the $p$-norms was no small matter. In the case of (1.2), the relevant cone functions are of the form

$$
u(x)=a \pm\|x-z\|_{*} .
$$

The results we establish for (1.2) apply immediately to equations $H(D u)=1$ where

$$
H \in C^{2}\left(\mathcal{R}^{n}\right), H(p) \geq 0, H(0)=0, H(p)=H(-p),
$$

and

$$
\left\langle D^{2} H(p) \xi, \xi\right\rangle \geq \kappa|\xi|^{2} \text { for } p, \xi \in \mathcal{R}^{n}
$$

for some $\kappa>0$. Here $D^{2} H$ denotes the Hessian matrix of $H$. For this purpose one defines $\|\cdot\|$ by setting its unit sphere to be $\{p: H(p)=1\}$. See Remark 4.5 below. In this case we have verified for ourselves that the symmetry condition $H(p)=H(-p)$ in (1.6) can be dropped, which results in $\|\cdot\|$ not necessarily being origin symmetric. However, it becomes too distracting to live without the origin symmetric property of norms throughout this work and the companion paper Caffarelli and Crandall [2], upon which we will often call, although this should be a straightforward if tedious matter.

For any nonempty subset $A$ of $\mathcal{R}^{n}$, the distance from $x$ to $A$ is measured in $\|\cdot\|_{*}$ and is denoted by

$$
d(x, A):=\inf _{y \in A}\|x-y\|_{*} .
$$


A key ingredient in the proofs is the "distance formula":

$$
\begin{array}{lll}
u(x)=r+d\left(x, L_{r}\right) & \text { if } & u(x) \geq r, \\
u(x)=r-d\left(x, L_{r}\right) & \text { if } & u(x) \leq r .
\end{array}
$$

This is to hold when $L_{r}$ is a nonempty regular level set of $u$. That is,

$$
L_{r}:=\left\{x \in \mathcal{R}^{n}: u(x)=r\right\}
$$

is nonempty and $L_{r} \cap \mathcal{S}=\emptyset$. We could have presented verification of (1.9) as the main thrust of this paper and described the results explained before as an application of (1.9), but perhaps the current spin is more catchy. Our results concerning the validity of (1.9) go beyond what is needed to establish the consequences explained so far.

In Section 2 we set notation, some definitions, and provide some preliminary results. In Section 3 we discuss the distance formula and establish consequences of it. This leaves to the last section, Section 4, the verification of the distance formula in various cases. The crown jewel of this last section is Theorem 4.9, which covers all the $p$-norms (1.4), $1<p<\infty$, and which, together with the results of Section 3, establishes

the initial claims above for these cases and many others. Results from [2] needed herein are formatted for the current purpose in Appendix A.

\section{Notation, Definitions and Preliminaries}

The following notations used in the introduction will be used throughout this paper: $\langle x, y\rangle$ is the Euclidean inner-product of $x$ and $y,|x|$ is the Euclidean norm of $x,\|\cdot\|$ is a norm on $\mathcal{R}^{n}$ and $\|\cdot\|_{*}$ is its dual, as given by (1.3). We will use without comment the relation

$$
\langle x, y\rangle \leq\|x\|\|y\|_{*},
$$

which follows immediately from the definition of $\|y\|_{*}$.

Next, $d(x, A)$ is given by (1.8) and $\mathcal{S}$ will denote a closed subset of $\mathcal{R}^{n}$ with further properties that will vary from place to place in the proceedings.

Unless otherwise said, it is assumed that

$$
\|\cdot\| \quad \text { is strictly convex and differentiable off the origin. }
$$

We take as known that (2.1) implies the same properties for $\|\cdot\|_{*}$.

Unadorned balls will be defined by $\|\cdot\|_{*}$ :

$$
B_{r}(x):=\left\{y \in \mathcal{R}^{n}:\|x-y\|_{*}<r\right\}, \bar{B}_{r}(x):=\left\{y \in \mathcal{R}^{n}:\|x-y\|_{*} \leq r\right\}
$$

The unit spheres in $\|\cdot\|$ and $\|\cdot\|_{*}$ will be denoted as follows:

$$
S:=\left\{x \in \mathcal{R}^{n}:\|x\|=1\right\}, S^{*}:=\left\{x \in \mathcal{R}^{n}:\|x\|_{*}=1\right\} .
$$

We rely on the alert reader to distinguish between $S$ and $\mathcal{S}$. 
Remark 2.1. If $\|\cdot\|$ is not strictly convex, then $S$ contains a nontrivial line segment $[z, w]$ (see (2.5)). Let $g: \mathcal{R} \rightarrow[0,1]$ be continuously differentiable and satisfy $0 \leq g^{\prime}(t) \leq 1$ for $t \in \mathcal{R}$. Define

$$
u(x)=g(\langle x, z-w\rangle)+\langle x, w\rangle .
$$

Then $u \in C^{1}\left(\mathcal{R}^{n}\right)$ and

$$
D u(x)=g^{\prime}(\langle x, z-w\rangle)(z-w)+w=g^{\prime}(\langle x, z-w\rangle) z+\left(1-g^{\prime}(\langle x, z-w\rangle) w \in[z, w],\right.
$$

so $\|D u\|=1$. However, $u$ is not affine if $g$ is not constant. Thus strict convexity is necessary for the assertions in the introduction to hold for $\|\cdot\|$. We do not have an example documenting the necessity that $\|\cdot\|$ be differentiable, although this assumption is firmly used in arguments below.

Euclidean balls will be denoted as follows:

$$
\begin{aligned}
& B_{r}^{E}(x):=\left\{y \in \mathcal{R}^{n}:|x-y|<r\right\}, \\
& \bar{B}_{r}^{E}(x):=\left\{y \in \mathcal{R}^{n}:|x-y| \leq r\right\} .
\end{aligned}
$$

We will not need a notation for balls defined by $\|\cdot\|$.

The line segment from $y$ to $x$ is denoted by

$$
[y, x]:=\{(1-t) y+t x: 0 \leq t \leq 1\} .
$$

If we want to parameterize $[y, x]$, we write

$$
[y, x]_{t}:=(1-t) y+t x .
$$

We will sometimes regard $[y, x]$ as directed (it has an initial point $y$ and terminal point $x$ ), and sometimes merely as a set, as would be the case if we wrote $z \in[y, x]$. The context will make it clear which notion is in play. The anti-alphabetical ordering in $[y, x]$ employed here is for consistency with later usages.

We will write and have written $A:=B$ to emphasize that $A$ is defined to be $B$. We will also write

$$
x_{j} \rightarrow x
$$

to indicate that $x_{j}$ is a sequence of elements of $\mathcal{R}^{n}$ convergent to $x$. The context will make clear whether $x_{j}$ is a vector, as above, or a coordinate, as in (1.4).

We next review some elementary facts. As $\|\cdot\|$ is differentiable, we may define

$$
N(x):=D\|x\|
$$

at each $x \neq 0$. Here and elsewhere $D v(x)$ denotes the gradient of the function $v$ at any point $x$ where $v$ is differentiable. Clearly $N(x)$ is an exterior normal to $\|x\| S$ at $x$; equivalently, it is an exterior normal to $S$ at $x /\|x\|$.

We take as known that $N$ is continuous on its domain. Since the norm is positive homogeneous of degree 1 and even, $N$ is positive homogeneous of degree 0 and odd, or

$$
N(\lambda x)=N(x), N(-x)=-N(x) \text { for } \quad x \neq 0, \lambda>0 .
$$


We take as known that $N$ has the properties (2.9) below and that they, in fact, characterize $N(x)$ :

$$
\langle x, N(x)\rangle=\|x\|,\|N(x)\|_{*}=1 \quad \text { for } \quad x \neq 0 .
$$

Finally, since $\|\cdot\|$ is strictly convex,

$$
N: S \rightarrow S^{*} \text { is one-to-one. }
$$

See [2] for further elementary discussion of these standard facts.

Since $\|\cdot\|_{*}$ is also strictly convex and differentiable,

$$
N^{*}(x):=D\|x\|_{*}
$$

shares the properties of $N$ with $\|\cdot\|$ and $\|\cdot\|_{*}$ interchanged. Here we are taking as known that $\left(\|\cdot\|_{*}\right)_{*}=\|\cdot\|$; this is true whenever $\|\cdot\|$ is a norm, but takes some proving. In our situation, it follows immediately from (2.9). Moreover, from

$$
\left\langle N^{*}(x), N\left(N^{*}(x)\right)\right\rangle=\left\|N^{*}(x)\right\|=1=\left\|N\left(N^{*}(x)\right)\right\|_{*}
$$

and the characterization (2.9) applied $N^{*}(x)$, we see that $N^{*}(x)=N^{*}\left(N\left(N^{*}(x)\right)\right.$. Since $N^{*}: S^{*} \rightarrow S$ is one-to-one, it follows that

$$
N^{*}: S^{*} \rightarrow S \quad \text { and } \quad N: S \rightarrow S^{*} \text { are inverse to each other. }
$$

The function $u$ will always satisfy

$$
u: \mathcal{R}^{n} \backslash \mathcal{S} \rightarrow \mathcal{R} \quad \text { is differentiable at each point of } \mathcal{R}^{n} \backslash \mathcal{S},
$$

and

$$
\|D u(x)\|=1 \quad \text { for } \quad x \in \mathcal{R}^{n} \backslash \mathcal{S} .
$$

Note that, by the discussion of $N, N^{*}, u(x)=\|x\|_{*}$ satisfies (2.14) with $\mathcal{S}=\{0\}$.

Throughout this paper it will be assumed that

$$
\text { the Hausdorff }(n-1) \text {-measure of } \mathcal{S} \text { is } 0 .
$$

For brevity, we might say instead that "the area of $\mathcal{S}$ is 0 ," meaning that (2.15) holds. These are minimal assumptions for us, and we see next some implications of them.

The assertion (2.18) of Lemma 2.2 below would be immediate from the method of characteristics if sufficient regularity was assumed of $\|\cdot\|$ and $v$. Here we do not even assume that $v$ is $C^{1}$, instead it just pointwise differentiable, as with $u$ above. This is not an important point, as the lemma states that then $v$ is $C^{1}$ anyway. Since we will need (2.18) in nonclassical generality, we may as well start with minimal assumptions on $v$ as well. An alternative to the elementary self-contained proof we present would be to quote some results in the literature, which, however, requires more knowledge of the reader to peruse. This alternative is discussed in Remark 2.3 below.

Lemma 2.2. Let $U$ be an open subset of $\mathcal{R}^{n}$ and $v: U \rightarrow \mathcal{R}$ be differentiable at each point of $U$ and satisfy

$$
\|D v(x)\|=1 \quad \text { for } \quad x \in U .
$$


Suppose that $x_{0} \in U, a \leq 0 \leq b, \mathcal{I}=(a, b)$ and

$$
x_{0}+t N\left(D v\left(x_{0}\right)\right) \in U \quad \text { for } \quad t \in \mathcal{I} .
$$

Then for $t \in \mathcal{I}$

$$
\begin{aligned}
& v\left(x_{0}+t N\left(D v\left(x_{0}\right)\right)=v\left(x_{0}\right)+t\right. \text { and } \\
& D v\left(x_{0}+t N\left(D v\left(x_{0}\right)\right)=D v\left(x_{0}\right) .\right.
\end{aligned}
$$

Moreover, $v \in C^{1}(U)$.

Proof. Let $\bar{B}_{R}\left(x_{0}\right) \subset U$. It suffices to show that (2.18) holds for $|t| \leq R$; the general result then follows from a simple continuation argument.

Let

$$
M_{r}=\max _{\bar{B}_{r}\left(x_{0}\right)} v, m_{r}=\min _{\bar{B}_{r}\left(x_{0}\right)} v \quad \text { for } \quad 0 \leq r \leq R
$$

We claim that

$$
M_{r}=v\left(x_{0}\right)+r, m_{r}=v\left(x_{0}\right)-r .
$$

We use (2.9) freely in the discussion.

First, suppose that $x, y \in \bar{B}_{R}\left(x_{0}\right)$. Then

$$
\begin{aligned}
\left|\frac{d}{d t} v(x+t(y-x))\right| & =|\langle D v(x+t(y-x)), y-x\rangle| \\
& \leq\|D v(x+t(y-x))\|\|y-x\|_{*}=\|x-y\|_{*} .
\end{aligned}
$$

It follows that

$$
|v(y)-v(x)| \leq\|y-x\|_{*}
$$

and from this that $r \mapsto M_{r}$ has 1 as a Lipschitz constant. On the other hand, for $0 \leq r<R$, let $x_{r}^{+} \in \bar{B}_{r}\left(x_{0}\right)$ satisfy $v\left(x_{r}^{+}\right)=M_{r}$. Then for small $h>0$

$$
\begin{aligned}
\frac{M_{r+h}-M_{r}}{h} & \geq \frac{v\left(x_{r}^{+}+h N\left(D v\left(x_{r}^{+}\right)\right)\right)-v\left(x_{r}^{+}\right)}{h} \\
& =\left\langle D v\left(x_{r}^{+}\right), N\left(D v\left(x_{r}^{+}\right)\right)\right\rangle+\mathrm{o}(1) \\
& =\left\|D v\left(x_{r}^{+}\right)\right\|+\mathrm{o}(1)=1+\mathrm{o}(1)
\end{aligned}
$$

where o(1) denotes a quantity tending to 0 as $h \downarrow 0$. Thus $d M_{r} / d r=1$ a.e. and the first assertion of (2.19) follows. The second assertion follows upon applying the first to $-v$.

Now let $x_{R}^{-} \in \bar{B}_{R}\left(x_{0}\right)$ be such that $v\left(x_{R}^{-}\right)=m_{R}=v\left(x_{0}\right)-R$. Since we have (2.20),

$$
v\left(x_{R}^{+}\right)-v\left(x_{R}^{-}\right)=2 R \text { and }\left\|x_{R}^{+}-x_{R}^{-}\right\| \leq 2 R,
$$

it must be that $\left\|x_{R}^{+}-x_{R}^{-}\right\|=2 R$. Since $\|\cdot\|_{*}$ is strictly convex, $x_{R}^{+}, x_{R}^{-}$are necessarily antipodal points of the sphere $\partial B_{R}\left(x_{0}\right)$. This implies that $x_{R}^{+}$and $x_{R}^{-}$are unique, for any other point of maximum must be antipodal to $x_{R}^{-}$, etc. 
Now put

$$
e^{*}=\frac{x_{R}^{+}-x_{0}}{R}
$$

Then $\left\|e^{*}\right\|_{*}=1$. For $0 \leq t \leq R$ define

$$
g(t)=v\left(x_{0}+t e^{*}\right)-v\left(x_{0}\right)
$$

By (2.20), $\left|g^{\prime}(t)\right| \leq 1$, while $g(0)=0, g(R)=R$. It follows that $g(t)=t$, and then

$$
1=g^{\prime}(t)=\left\langle D v\left(x_{0}+t e^{*}\right), e^{*}\right\rangle \quad \text { for } \quad 0 \leq t<R .
$$

The characterization $(2.9)$ of $N(x)$ then implies that

$$
e^{*}=N\left(D v\left(x_{0}+t e^{*}\right)\right) \quad \text { for } \quad 0 \leq t \leq R
$$

in particular,

$$
e^{*}=N\left(D v\left(x_{0}\right)\right) .
$$

However, $N$ is one-to-one on $S$ because $\|\cdot\|$ is strictly convex. Therefore we have

$$
D v\left(x_{0}+t N\left(D v\left(x_{0}\right)\right)\right)=D v\left(x_{0}\right) \quad \text { for } \quad 0 \leq t \leq R
$$

which, together with the above, establishes (2.18) for $0 \leq t \leq R$. We may discuss negative $t$ in a similar manner and (2.18) is proved.

To see that $D v$ is continuous, let $x_{j} \rightarrow x_{0} \in U$ and $\bar{B}_{R}\left(x_{j}\right) \subset U$ for large $j$. By what was already shown,

$$
v\left(x_{j}+R N\left(D v\left(x_{j}\right)\right)\right)=v\left(x_{j}\right)+R .
$$

Passing to a subsequence if neccessary, we may assume that $D v\left(x_{j}\right) \rightarrow \hat{e}$ for some $\hat{e} \in S$. Passing to the limit in the relation above, we then find

$$
v\left(x_{0}+R N(\hat{e})\right)=v\left(x_{0}\right)+R ;
$$

that is, $x_{0}+R N(\hat{e})$ is a point of maximum of $v$ in $\bar{B}_{R}\left(x_{0}\right)$. By the uniqueness of points of maximum, already argued, we conclude that $N(\hat{e})=N\left(D v\left(x_{0}\right)\right)$ and then $\hat{e}=D v\left(x_{0}\right)$. The continuity of $D v$ follows.

Remark 2.3. Since $v$ is assumed differentiable everywhere on $U$, it is a viscosity solution of both $\|D v\|-1=0$ and $-\|D v\|+1=0$. By Proposition 5.3.4 of Cannarsa and Sinestrari [3], which generalizes results first proved by Lions [13], $v$ is then both semiconvex and semiconcave in the sense of [3]. Then by Theorem 3.3.7 of [3], $v$ is $C^{1}$; however, this is a long road to this result. Moreover, once $x_{R}^{ \pm}$are in hand, via the proof we gave, the second relation of (2.18), and hence the first, follows from Lemma 7.1 of [6], whose proof is a generalization of an argument of Aronsson [1]. See also Fitzpatrick [9]. Moreover, it also follows from the cited lemma of [6] that if $U=\mathcal{R}^{n}$, then $v$ is affine. This last result is not entirely new. It is proved in the case of the Euclidean norm and analytic $v$ in D. Khavinson [11]. Another instance of "tight on a line implies affine" appeared in Crandall and Evans [7] in the Euclidean case. See also Proposition 3.3.

According to the next lemma, $u$ may be extended to $\mathcal{S}$ by continuity. The reader is invited to skip the proof. 
Lemma 2.4. Let u satisfy (2.13) and (2.14) and $\mathcal{S}$ have 0 area. Then $u$ extends by continuity to all of $\mathcal{R}^{n}$, and the extension by continuity, still denoted by $u$, satisfies

$$
|u(x)-u(y)| \leq\|x-y\|_{*} \quad \text { for } \quad x, y \in \mathcal{R}^{n} .
$$

Proof. It is enough to verify (2.21) for $x, y \in \mathcal{R}^{n} \backslash \mathcal{S}$, which is dense in $\mathcal{R}^{n}$ under our assumptions. Let $x, y \in \mathcal{R}^{n} \backslash \mathcal{S}$ and let $\gamma:[0, T] \rightarrow \mathcal{R}^{n} \backslash \mathcal{S}$ be such that $\gamma(0)=x, \gamma(T)=y$. Then

$$
u(y)-u(x)=\int_{0}^{T} \frac{d}{d t} u(\gamma(t)) d t=\int_{0}^{T}\langle D u(\gamma(t)), \dot{\gamma}(t)\rangle d t \leq \int_{0}^{T}\|\dot{\gamma}(t)\|_{*} d t .
$$

Thus the result follows if for each $\epsilon>0$ there is a $\gamma$ with the additional property that its length (measured in $\|\cdot\|_{*}$ ) satisfies

$$
\operatorname{length}(\gamma)=\int_{0}^{T}\|\dot{\gamma}(t)\|_{*} d t \leq\|x-y\|_{*}+\epsilon .
$$

To this end, let $\kappa>0$ be such that

$$
\bar{B}_{\kappa}^{E}(w) \cap \mathcal{S}=\emptyset \text { for } \quad w=x, y .
$$

This is possible since $x, y \in \mathcal{R}^{n} \backslash \mathcal{S}$, which is open. We claim that there is a sequence of points $z_{j} \in$ $\partial B_{|x-y|}^{E}(x) \cap B_{\kappa}^{E}(y)$ such that $z_{j} \rightarrow y$ and $\left[z_{j}, x\right] \cap \mathcal{S}=\emptyset$. This proves the claim, once established, for then the path $\gamma:[0,2] \rightarrow \mathcal{R}^{n}$ defined by

$$
\gamma(t)=\left\{\begin{array}{l}
{\left[x, z_{j}\right]_{t} \text { for } \quad 0 \leq t \leq 1,} \\
{\left[z_{j}, y\right]_{t-1} \text { for } \quad 1 \leq t \leq 2,}
\end{array}\right.
$$

does not meet $\mathcal{S}$ and has length $\left\|x-z_{j}\right\|_{*}+\left\|z_{j}-y\right\|_{*} \rightarrow\|x-y\|_{*}$.

Let $|x-z|=|x-y|$; then for $0 \leq t \leq 1$,

$$
\left|x-[x, z]_{t}\right|=t|x-z|=t|x-y|
$$

and so $[x, z]_{t} \notin \mathcal{S}$ for $t \leq \kappa /|x-y|$ by $(2.23)$.

Let

$$
A=\left\{[x, z]_{t}:|x-z|=|x-y|, \kappa /|x-y| \leq t \leq 1\right\} .
$$

It is proved in [2] (see also Remark 4.5) that

$$
A \ni[x, z]_{t} \mapsto z
$$

is well defined and Lipschitz continuous. In (2.24) $z, t$ are subject to the restrictions which appear in the definition of $A$. If for each choice of $z \in B_{1 / j}^{E}(y) \cap \partial B_{|x-y|}^{E}(x)$, which is a piece of surface of positive area, the line segment $[x, z]$ meets $\mathcal{S}$, then $B_{1 / j}^{E}(y) \cap \partial B_{|x-y|}^{E}(x)$ would be the Lipschitz image of a subset of $A \cap \mathcal{S}$, which has 0 area, under the map (2.24). This is a contradiction. Thus for each large enough $j=1,2, \ldots$, there is a $z_{j} \in B_{1 / j}^{E}(y) \cap \partial B_{|x-y|}^{E}(x)$ for which $\left[x, z_{j}\right] \cap \mathcal{S}=\emptyset$.

In view of this lemma, hereafter $u$ is regarded as defined on $\mathcal{R}^{n}$ and its level sets are denoted by

$$
L_{r}:=\left\{x \in \mathcal{R}^{n}: u(x)=r\right\}=:\{u=r\},
$$


wherein we are also announcing notations such as $\{u=r\}$ above, and

$$
\{r<u<R\}:=\left\{x \in \mathcal{R}^{n}: r<u(x)<R\right\} .
$$

\section{The Distance Formula and Its Consequences}

In this section, $u, \mathcal{S}$ always satisfy the assumptions of Section 2 and we recall that we regard $u$ as defined on $\mathcal{R}^{n}$. In the next section, we verify the relations

$$
\begin{array}{lll}
u(x)=r+d\left(x, L_{r}\right) & \text { if } & u(x) \geq r, \\
u(x)=r-d\left(x, L_{r}\right) & \text { if } & u(x) \leq r,
\end{array}
$$

which we call the "distance formula," under the assumptions that $L_{r}$ is nonempty and

$$
L_{r} \cap \mathcal{S}=\emptyset
$$

in various circumstances. If $L_{r}$ is nonempty and satisfies (3.2) we simply say that " $L_{r}$ is a regular level set of $u$ " or " $r$ is a regular value of $u . "$

In the current section, we observe in Lemma 3.1 that the set of $r \in u\left(\mathcal{R}^{n}\right)$ for which the distance formula holds is closed in $u\left(\mathcal{R}^{n}\right)$, and in Remark 3.2 that regular values of $u$ are dense in $u\left(\mathcal{R}^{n}\right)$ if $\mathcal{S}$ has 0 length. Then we establish some consequences of the distance formula in Proposition 3.3. Among the results of Section 4 below, is that the distance formula holds for regular values of $u$ when $\|\cdot\|$ is any $p$-norm, $1<p<\infty$, and $\mathcal{S}$ has 0 length. The initial claims in the introduction follow from this and Proposition 3.3 in these cases.

Lemma 3.1. Let $x \in \mathcal{R}^{n}$. Then the set of values of $r$ for which (3.1) holds is closed in $u\left(\mathcal{R}^{n}\right)$.

Proof. Suppose that $r_{j} \in u\left(\mathcal{R}^{n}\right), r_{j} \rightarrow R \in u\left(\mathcal{R}^{n}\right)$ and (3.1) holds for $r=r_{j}, j=1,2, \ldots$ We show that then (3.1) holds for $r=R$. Assume that $u(x)>R$. Then $u(x)>r_{j}$ for large $j$ and, by assumption,

$$
u(x)=r_{j}+d\left(x, L_{r_{j}}\right)=r_{j}+\left\|x-y_{j}\right\|_{*}
$$

where $y_{j} \in L_{r_{j}}$ is a nearest point to $x$. Clearly $y_{j}$ is bounded, so we may assume that $y_{j} \rightarrow y$. Since $u\left(y_{j}\right)=r_{j}, u(y)=R$ and $y \in L_{R}$. Thus (3.3) implies that

$$
u(x)=R+\|x-y\|_{*} \geq R+d\left(x, L_{R}\right) .
$$

But we also have the reverse inequality, and hence equality. Indeed, if $z \in L_{R}$ is a nearest point to $x$, then

$$
u(x)-R=u(x)-u(z) \leq\|x-z\|_{*}=d\left(x, L_{R}\right) .
$$

Remark 3.2. If we assume that $\mathcal{S}$ has 0 length, then, owing to the Lipschitz continuity of $u$, so does $u(\mathcal{S})$. But $u\left(\mathcal{R}^{n}\right)$ is a nontrivial interval, so, in this case, the regular values of $u$ are dense in $u\left(\mathcal{R}^{n}\right)$.

In the following proposition

$$
M:=\sup _{\mathcal{R}^{n}} u, m:=\inf _{\mathcal{R}^{n}} u
$$


Proposition 3.3. Let the distance formula hold for all $x \in \mathcal{R}^{n}$ and every nonempty level set $L_{r}$ of $u$. Then the following assertions hold.

(i) $u$ is $C^{1}$ on the set $\{x: m<u(x)<M\}$.

(ii) If $M=\infty$, then $u$ is convex, and if $m=-\infty$, then $u$ is concave.

(iii) If $M, m$ are both infinite, then $u$ is affine.

(iv) If $\mathcal{S}$ has 0 length, $-\infty<m$ and $M=\infty$ (respectively, $-\infty=m$ and $M<\infty)$, then $u(x)=m+\|x-z\|_{*}$ (respectively, $u(x)=M-\|x-z\|_{*}$ ) for some $z \in \mathcal{R}^{n}$.

(v) If $\mathcal{S}$ has 0 length and $n \geq 2$, then $u$ is not bounded, so at least one of $M, m$ is infinite.

We prepare a lemma which will immediately yield (i) above. Moreover, elements of its proof are used to establish (ii) (and hence (iii)). The lemma only requires $\|\cdot\|_{*}$ to be differentiable off the origin (equivalently, $\|\cdot\|$ to be strictly convex).

Lemma 3.4. Let $A, B \subset \mathcal{R}^{n}$ be nonempty and closed and $U \subset \mathcal{R}^{n} \backslash(A \cup B)$ be nonempty and open. Suppose that $\|\cdot\|_{*}$ is differentiable off the origin and

$$
d(x, A)+d(x, B)=c \quad \text { for } \quad x \in U
$$

where $c$ is some constant. Then $x \mapsto d(x, A)$ is continuously differentiable on $U$.

Proof. To see this, we first note that $d(x, A)$ has a "superdifferential" (here we just mean that (3.8) below holds) at each point $x \notin A$. Indeed, if $x \notin A, z \in A$, and $\|y\|_{*}<d(x, A)$, we have

$$
\|x-z+y\|_{*}-\|x-z\|_{*}=\left\langle y, N^{*}(x-z)\right\rangle+\int_{0}^{1}\left\langle y, N^{*}(x-z+t y)-N^{*}(x-z)\right\rangle d t .
$$

Therefore, choosing $z_{A} \in A$ so that $\left\|x-z_{A}\right\|_{*}=d(x, A)$ and using $d(x+y, A) \leq\left\|x-z_{A}+y\right\|_{*}$, we find

$$
d(x+y, A)-d(x, A) \leq\left\langle y, N^{*}\left(x-z_{A}\right)\right\rangle+\int_{0}^{1}\left\langle y, N^{*}\left(x-z_{A}+t y\right)-N^{*}\left(x-z_{A}\right)\right\rangle d t .
$$

We will also use the modulus of continuity of $N^{*}$. Since $N^{*}$ is continuous on the complement of the origin, there is a nondecreasing function $\rho:[0, \infty) \rightarrow[0,2]$ satisfying $\rho(0+)=0$ such that

$$
\left\|N^{*}(x+h)-N^{*}(x)\right\| \leq \rho\left(\|h\|_{*}\right) \quad \text { for } \quad\|x\|_{*}=1, x+h \neq 0 .
$$

Note that the left hand side of above, when defined (i.e., $x+h \neq 0$ ), is never more than 2. Next, via homogeneity,

$$
\left\|N^{*}(x+h)-N^{*}(x)\right\| \leq \rho\left(\frac{\|h\|_{*}}{\|x\|_{*}}\right) \quad \text { for } \quad x, x+h \neq 0 .
$$

Using this in (3.6) we find

$$
d(x+y, A)-d(x, A) \leq\left\langle y, N^{*}\left(x-z_{A}\right)\right\rangle+\|y\|_{*} \rho\left(\frac{\|y\|_{*}}{d(x, A)}\right) .
$$


Letting $z_{B} \in B$ be such that $\left\|x-z_{B}\right\|_{*}=d(x, B)$, we have the estimate (3.8) with $A$ replaced everywhere by $B$. Combining this with (3.5) results in

$$
\begin{aligned}
-\left(\left\langle y, N^{*}\left(x-z_{B}\right)\right\rangle+\|y\|_{*} \rho\left(\frac{\|y\|_{*}}{d(x, B)}\right)\right) \leq & -(d(x+y, B)-d(x, B))=d(x+y, A)-d(x, A) \\
& \leq\left\langle y, N^{*}\left(x-z_{A}\right)\right\rangle+\|y\|_{*} \rho\left(\frac{\|y\|_{*}}{d(x, A)}\right) .
\end{aligned}
$$

Because $\rho(0+)=0$, the nonlinear terms in $y$ in the extremes of this series of relations are $\mathrm{o}\left(\|y\|_{*}\right)$, and therefore the linear terms must agree, that is

$$
N^{*}\left(x-z_{A}\right)=-N^{*}\left(x-z_{B}\right) .
$$

It follows that $N^{*}\left(x-z_{A}\right)$ and $N^{*}\left(x-z_{B}\right)$ do not depend on the choices of $z_{A}$ and $z_{B}$, should there be more than one, and

$$
\operatorname{Dd}(x, A)=-D d(x, B)=N^{*}\left(x-z_{A}\right)=-N^{*}\left(x-z_{B}\right) .
$$

The continuity of $\operatorname{Dd}(x, A)$ also follows. If $x_{j} \rightarrow x$ and $z_{A, j}$ is a nearest point in $A$ to $x_{j}$, we may assume that $z_{A, j} \rightarrow z_{A}$, a nearest point to $x$. By continuity of $N^{*}$ and the above, we then have

$$
\operatorname{Dd}\left(x_{j}, A\right)=N^{*}\left(x_{j}-z_{A, j}\right) \rightarrow N^{*}\left(x-z_{A}\right) .
$$

Remark 3.5. Proposition 2.1.2 and Proposition 2.1.5 of [3] combine to imply that $x \mapsto d(x, A)$ is locally semiconcave off of $A$ in the sense of [3]. Our proof follows the standard path to such results, but provides details we use below. The assumption (3.5) then implies that $d(x, A)$ is locally semiconcave and semiconvex on $U$, and therefore $C^{1}$ on $U$, by Theorem 3.3 .7 of [3]. This is a very nice theorem, but, as said once before, the road to it is long compared to the simple, direct, proof above for our special case.

Proof of Proposition 3.3. Choose $R, r \in u\left(\mathcal{R}^{n}\right)$ such that $m<r<R<M$. Note that, by assumption,

$$
u(x)=r+d\left(x, L_{r}\right)=R-d\left(x, L_{R}\right)
$$

on $U:=\{r<u<R\}$, so $d\left(x, L_{r}\right)+d\left(x, L_{R}\right)=R-r$ on $U$. Applying Lemma 3.4 with $A=L_{r}, B=L_{R}$, we see that $u$ is $C^{1}$ on $U$. This proves (i).

Suppose now that $m=-\infty$. Suppose also that $r<u(x), u(x \pm y)$. Use the first equality in (3.10) at $x, x+y$ and then (3.8) (with $A=L_{r}$ ) to conclude that

$$
\begin{aligned}
u(x+y)-u(x) & =d\left(x+y, L_{r}\right)-d\left(x, L_{r}\right) \\
& \leq\left\langle y, N^{*}\left(x-z_{r}\right)\right\rangle+\|y\|_{*} \rho\left(\frac{\|y\|_{*}}{d\left(x, L_{r}\right)}\right),
\end{aligned}
$$

where $z_{r} \in L_{r}$ is a nearest point to $x$. Write the same inequality with $y$ replaced by $-y$ and add to conclude that

$$
u(x+y)+u(x-y)-2 u(x) \leq 2\|y\|_{*} \rho\left(\frac{\|y\|_{*}}{d\left(x, L_{r}\right)}\right) .
$$


Now let $r \downarrow-\infty$, which entails $d\left(x, L_{r}\right) \rightarrow \infty$. By $\rho(0+)=0$, the right hand side above then tends to 0 , and we conclude that $u$ is concave; the case $M=\infty$ is similar (or replace $u$ by $-u$ ). This proves (ii). The assertion (iii) follows at once from (ii).

To prove (iv), we note that if $-\infty<m$, then $L_{m}$ is not empty. Indeed, by (i) and Lemma 2.2, for any $x$, $m<u(x)$, we have

$$
u(x-t N(D u(x))=u(x)-t
$$

for $t>0$, so long as $x-t N(D u(x))$ stays in the set $\{-m<u<M\}$. By continuity,

$u(x-(u(x)-m) N(D u(x)))=m$. If $M=\infty, u$ is convex by (ii), and the set of its minimizing points, $L_{m}$, is convex. Since $L_{m} \subset \mathcal{S}$, which has 0 length, $L_{m}$ must be a single point, say $L_{m}=\{z\}$. Then by the distance formula

$$
u(x)=m+d\left(x, L_{m}\right)=m+\|x-z\|_{*} .
$$

Treating the other case similarly, this proves (iv).

Finally, to prove (v), assume $m, M$ are both finite. Then $L_{m}, L_{M}$ are nonempty. Let $u(x)=(M+m) / 2$. Then

$$
m=u\left(x-\frac{M-m}{2} N(D u(x))\right), M=u\left(x+\frac{M+m}{2} N(D u(x))\right) .
$$

That is, the maps

$$
T_{M}(x)=x+\frac{M+m}{2} N(D u(x)), T_{m}(x)=x-\frac{M-m}{2} N(D u(x))
$$

satisfy

$$
T_{M}: L_{(M+m) / 2} \rightarrow L_{M}, T_{m}: L_{(M+m) / 2} \rightarrow L_{m} .
$$

Restricting these maps to an arcwise connected neighborhood of $x_{0}$ in $L_{(M+m) / 2}$, the images of this neighborhood are arcwise connected. Since $L_{m}, L_{M}$ have length 0, the images must be singletons. However, clearly, if $x \neq \hat{x}$, and $T_{m}(x)=T_{m}(\hat{x})$, then $T_{M}(x) \neq T_{M}(\hat{x})$, etc. This yields a contradiction.

\section{Verification of the Distance Formula}

We turn to the issue of verifying (3.1) for regular values $r$ of $u$, beginning with a simple lemma.

Lemma 4.1. Suppose that $L_{r}$ is a regular level set of $u, x \in \mathcal{R}^{n}, y \in L_{r}$, and

$$
d\left(x, L_{r}\right)=\|x-y\|_{*} .
$$

Suppose further that $[y, x] \cap \mathcal{S}=\emptyset$. Then the distance formula holds.

Proof. It suffices to treat the case $u(x) \neq r$, so $y \neq x$. Since $y \in L_{r}$ minimizes

$$
L_{r} \ni z \mapsto\|z-x\|_{*},
$$

and $D u(y)$ is normal to $L_{r}$ at $y$, Lagrange multipliers tell us that there is a $\lambda$ such that

$$
\left.D_{z}\|z-x\|_{*}\right|_{z=y}=N^{*}(y-x)=\lambda D u(y) .
$$


Since $D u(y)$ and $N^{*}(y-x)$ are both $\|\cdot\|$ unit vectors, $|\lambda|=1$. Moreover, it follows from (2.8), (2.12) and homogeneity that

$$
\frac{y-x}{\|y-x\|_{*}}=N(\lambda D u(y))=\lambda N(D u(y)) \text {. }
$$

We have yet to decide whether $\lambda$ is 1 or -1 . However, since $[y, x]$ is free of points of $\mathcal{S},(2.18)$ of Lemma 2.2 and (4.2) imply that

$$
\begin{array}{r}
u(y+t(x-y))=u\left(y+t\|x-y\|_{*} \frac{x-y}{\|x-y\|_{*}}\right)=u\left(y-t \lambda\|x-y\|_{*} N(D u(y))\right)= \\
u(y)-t \lambda\|x-y\|_{*}=r-t \lambda d\left(x, L_{r}\right) .
\end{array}
$$

for $0 \leq t \leq 1$. Putting $t=1$, we find (3.1) (clearly $\lambda=1$ if $u(x)<r, \lambda=-1$ if $u(x)>r$ ).

We turn to the task of verifying the distance formula for all $x \in \mathcal{R}^{n}$ and regular level set $L_{r}$ in cases for which Lemma 4.1 does not directly apply. The results will involve an interaction between the size of $\mathcal{S}$, in the form that some Hausdorff measure of $\mathcal{S}$ is 0 , and properties of $\|\cdot\|_{*}$. We first sketch the general idea of the proof, and then describe the setting more precisely.

It follows from Lemma 4.1 that if the distance formula fails at $x_{0}$, then for each $y \in L_{r}$ for which $d\left(x_{0}, L_{r}\right)=\left\|x_{0}-y\right\|_{*},\left[y, x_{0}\right]$ must meet $\mathcal{S}$. Moreover, since $L_{r}$ is regular, it cannot be that $\left[y, x_{0}\right]_{t} \in \mathcal{S}$ if $0 \leq t$ is sufficiently small, and if the distance formula fails at $x_{0}$, then it fails in a neighborhood of $x_{0}$. To derive contradictions from these considerations, made precise, we will set up a mapping

$$
[y, x]_{t} \rightarrow x
$$

where $x$ ranges over a piece of the level set $d\left(x, L_{r}\right)=d\left(x_{0}, L_{r}\right), \mu \leq t \leq 1-\mu$ for some $0<\mu<1$ to be discussed, and $y \in L_{r}$ satisfies $d\left(x, L_{r}\right)=\|y-x\|_{*}$. Properties of this mapping established in [2] will allow us to derive contradictions, under various assumptions, to the assumption that each $[y, x]$ meets $\mathcal{S}$.

We make all this much more precise below, providing parameterizations of various ingredients. The discussion is somewhat rambling, as it provides a good part of the proofs of the results to follow. To conveniently call on it the platform it provides, we will the whole of it a name, the "Set Up". The Set Up continues until the header "The Set Up Has Ended" is found below.

\section{The Set Up}

Suppose the distance formula fails at $x_{0}$. Then it fails for all $x$ in some open neighborhood $\mathcal{N}$ of $x_{0}$. Since the points at which $x \mapsto d\left(x, L_{r}\right)$ is not differentiable are a set of Lebesgue measure 0 by the Lipschitz continuity of distance functions, and $\mathcal{S}$ has area 0 , we may choose $z \in \mathcal{N}$ such that $d\left(x, L_{r}\right)$ is differentiable at $z$ and $z \notin \mathcal{S}$. Replacing $x_{0}$ by $z$, we now assume that $x_{0} \notin \mathcal{S}, d\left(x, L_{r}\right)$ is differentiable at $x_{0}$, and the distance formula fails at all points in the neighborhood $\mathcal{N}$ of $x_{0}$.

As $d\left(x, L_{r}\right)$ is differentiable at $x_{0}$ and $\|\cdot\|_{*}$ is strictly convex, there is a unique $y_{0}$ satisfying

$$
y_{0} \in L_{r}, d\left(x_{0}, L_{r}\right)=\left\|x_{0}-y_{0}\right\|_{*},
$$


and then (see the proof of Lemma 3.4)

$$
\left.\operatorname{Dd}\left(x, L_{r}\right)\right|_{x=x_{0}}=N^{*}\left(x_{0}-y_{0}\right) .
$$

We take these simple facts as known. Similarly, if $d\left(x, L_{r}\right)$ is differentiable at $x_{j}$ and

$$
x_{j} \rightarrow x_{0}, y_{j} \in L_{r}, d\left(x_{j}, L_{r}\right)=\left\|x_{j}-y_{j}\right\|_{*},
$$

then

$$
\left.\operatorname{Dd}\left(x, L_{r}\right)\right|_{x=x_{j}}=\left.N^{*}\left(x_{j}-y_{j}\right) \rightarrow \operatorname{Dd}\left(x, L_{r}\right)\right|_{x=x_{0}} .
$$

because, by the uniqueness of $y_{0}, y_{j} \rightarrow y_{0}$.

We have merely recalled the fact that the gradient of $d\left(x, L_{r}\right)$ is continuous on the set of points of differentiability. We will need all the information in the following proposition concerning level sets of distance functions.

Proposition 4.2. Let $d\left(x, L_{r}\right)$ be differentiable at $x_{0} \notin L_{r}, y_{0} \in L_{r}$, and $d\left(x_{0}, L_{r}\right)=\left\|x_{0}-y_{0}\right\|_{*}$. Let

$$
\left.Z=\left\{z \in \mathcal{R}^{n}:\left\langle z, x_{0}-y_{0}\right)\right\rangle=0\right\} .
$$

Then there is a neighborhood $U$ of 0 in $Z$ and a neighborhood $V$ of $x_{0}$ in $\mathcal{R}^{n}$ and a Lipschitz continuous mapping $g: U \rightarrow \mathcal{R}$ such that $g(0)=0$ and if $s \in \mathcal{R}$ and

$$
z \in U, \text { and } x=x_{0}+s\left(x_{0}-y_{0}\right)+z \in V
$$

then

$$
d\left(x, L_{r}\right)=\left\|x_{0}-y_{0}\right\|_{*} \Longleftrightarrow s=g(z) .
$$

Proof. By the remarks preceding the proposition, the main result of F. H. Clarke in [5] applies to yield the claims concerning the existence of $g$, etc., once we notice that

$$
\begin{aligned}
\left.\frac{\partial}{\partial s} d\left(x_{0}+s\left(x_{0}-y_{0}\right)+z, L_{r}\right)\right|_{s=0, z=0} & =\left\langle\left. D d\left(x, L_{r}\right)\right|_{x=x_{0}}, x_{0}-y_{0}\right\rangle= \\
& \left\langle N^{*}\left(x_{0}-y_{0}\right), x_{0}-y_{0}\right\rangle=\left\|x_{0}-y_{0}\right\|_{*} \neq 0 .
\end{aligned}
$$

While the result in [5] is formulated as an inverse function theorem, one can deduce implicit function theorems from inverse function theorems in a standard way.

Remark 4.3. According to Proposition 4.2, near $x_{0}$ the level set

$$
L_{r}^{d}\left(x_{0}\right)=\left\{x: d\left(x, L_{r}\right)=d\left(x_{0}, L_{r}\right)=\left\|x_{0}-y_{0}\right\|_{*}\right\}
$$

of the distance function is the graph of a Lipschitz continuous function. Consequently, every neighborhood of $x_{0}$ in $L_{r}^{d}\left(x_{0}\right)$ has positive area.

We reprise and parameterize the above. If the distance formula fails, then it fails at some point $x_{0} \notin \mathcal{S}$ at which $d\left(x, L_{r}\right)$ is differentiable, and it fails at all points of the ball $\bar{B}_{3 \epsilon}\left(x_{0}\right)$ provided that $\epsilon>0$ is sufficiently 
small. Moreover, by choosing $\epsilon$ sufficiently small, we may assume that

$$
\bar{B}_{3 \epsilon}\left(x_{0}\right) \subset V, \bar{B}_{3 \epsilon}\left(x_{0}\right) \cap \mathcal{S}=\emptyset,
$$

where $V$ is from Proposition 4.2 and the second relation is due to $x_{0} \notin \mathcal{S}$, while $\mathcal{S}$ is closed.

Next, let $\nu>0$. The uniqueness of $y_{0}$ guarantees that if $\epsilon$ is sufficiently small and $x \in \bar{B}_{3 \epsilon}\left(x_{0}\right)$ and $y \in L_{r}$ is a nearest point to $x$, then $\left\|y-y_{0}\right\|_{*} \leq \nu$. That is

$$
x \in B_{3 \epsilon}\left(x_{0}\right), y \in L_{r},\|x-y\|_{*}=d\left(x, L_{r}\right) \Longrightarrow\left\|y-y_{0}\right\|_{*} \leq \nu .
$$

We assume that $\epsilon$ has been so chosen and that $\nu$ was also chosen so that

$$
\bar{B}_{3 \nu}\left(y_{0}\right) \cap \mathcal{S}=\emptyset .
$$

The set

$$
\Gamma:=\bar{B}_{\epsilon}\left(x_{0}\right) \cap\left\{x: d\left(x, L_{r}\right)=\left\|x_{0}-y_{0}\right\|_{*}=d\left(x_{0}, L_{r}\right)\right\},
$$

which is a graph of a Lipschitz continuous function by Proposition 4.2, will be of primary interest to us.

It will simplify the exposition here and later if we further assume that

$$
\left\|x_{0}-y_{0}\right\|_{*}=1 .
$$

This can be attained by appropriate scaling.

We note that if $x \in \Gamma$ and $y \in L_{r}$ is a nearest point to $x$, then

$$
[y, x]_{t} \notin \mathcal{S} \quad \text { for } \quad 0 \leq t \leq \nu \quad \text { and } \quad 1-\epsilon \leq t \leq 1 .
$$

The first assertion follows since

$$
\left\|[y, x]_{t}-y\right\|_{*}=t\|x-y\|_{*}=t \leq \nu
$$

implies

$$
\left\|[y, x]_{t}-y_{0}\right\|_{*} \leq\left\|[y, x]_{t}-y\right\|_{*}+\left\|y-y_{0}\right\|_{*} \leq 2 \nu
$$

and we have assumed (4.11). The second assertion follows in a similar manner from (4.9). Let $\mathcal{I}$ be the interval

$$
\mathcal{I}=[\nu, 1-\epsilon]
$$

note that if $\mathcal{I}$ is not a proper interval, then $[y, x] \cap \mathcal{S}=\emptyset$, and we are not concerned with this case. Define

$$
A=\left\{[y, x]_{t}: x \in \Gamma, y \in L_{r},\|x-y\|_{*}=1, t \in \mathcal{I}\right\}
$$

and optimistically attempt to define the "map" $F: A \rightarrow \Gamma$ by

$$
F\left([y, x]_{t}\right)=x,
$$

where the expression " $[y, x]_{t}$ " is subject to the conditions in the definition of elements of $A$. Indeed, from the introduction of [2], $F$ is well defined because $\|\cdot\|_{*}$ is strictly convex. 
Now, since the distance formula fails at all points of $\Gamma$, and we have (4.14) and Lemma 4.1, it follows that

$$
F(A \cap \mathcal{S})=\Gamma .
$$

Contradictions will be derived from this relation, Remark 4.3, which asserts that $\Gamma$ has positive area, and mapping properties of $F$ under various assumptions on $\mathcal{S}$ below.

\section{The Set Up Has Ended}

A quick sample result which we can now prove via the Set Up is this:

Proposition 4.4. Let $\|\cdot\|$ be the Euclidean norm. Let $L_{r}$ be a regular level set of $u$ and $\mathcal{S}$ have 0 area. Then the distance formula holds for $x \in \mathcal{R}^{n}$.

Proof. Assume that the distance formula does not hold at some $x_{0}$, and run the Set Up. According to Remark 4.5 below, $F$ is Lipschitz continuous on $A$. As the Lipschitz image of a set of 0 area has 0 area, (4.18) cannot hold, a contradiction.

Remark 4.5. The Lipschitz continuity used above also holds for the norm associated with (1.6). It is straightforward to see that this norm is "2-uniformly convex and 2-uniformly smooth," as explained in Appendix A, and then the dual norm shares these properties. Then one invokes Theorem A.2 with $p=q=2$. The Euclidean norm is a special case of this remark. However, treating the Euclidean norm in its own right, as is done in [2], the proof of Lipschitz continuity is especially clean, quick and simple.

In the cases $\|\cdot\|_{*}=\|\cdot\|_{p}, 1<p<\infty, p \neq 2$, it turns out that $F$ as used in the above proofs is not Lipschitz continuous, but it is Hölder continuous with exponent $2 / p$ if $p>2$ and with exponent $p / 2$ if $1<p<2$. See Remark A.1 and Theorem A.2. We consider these cases by assuming that $\alpha \in(0,1]$ and for every $0<\mu \leq 1$, $R>0$, there is a constant $C=C(\mu, R)$, such that

$$
\begin{gathered}
\mu \leq t \leq s \leq 1, \\
1=\|x-y\|_{*}=\|\hat{x}-\hat{y}\| \leq \min \left(\|x-\hat{y}\|_{*},\|\hat{x}-y\|_{*}\right)
\end{gathered}
$$

and

$$
\|x-\hat{x}\|_{*} \leq R,
$$

imply

$$
\|x-\hat{x}\|_{*} \leq C\left\|[y, x]_{t}-[\hat{y}, \hat{x}]_{s}\right\|_{*}^{\alpha} .
$$

Theorem A.2 provides examples.

Proposition 4.6. Let (4.22) hold when (4.19), (4.20), (4.21) hold. Let $L_{r}$ be a regular level set of $u$ and the Hausdorff $(n-1) \alpha$-measure of $\mathcal{S}$ be 0 . Then the distance formula holds for $x \in \mathcal{R}^{n}$.

Proof. Assume that the distance formula fails at some point. Run the Set Up. According to (4.22),

$$
\|F(z)-F(w)\|_{*} \leq C\|z-w\|_{*}^{\alpha}
$$


for some $C$. For any $\gamma>0$, we may cover $\mathcal{S}$ with a countable collection of balls of radius $r_{i}$ such that

$$
\mathcal{S} \subset \bigcup_{i=1}^{\infty} B_{r_{i}}\left(x_{i}\right), \sum_{i=1}^{\infty} r_{i}^{(n-1) \alpha} \leq \gamma
$$

Then

$$
\Gamma=F(A \cap \mathcal{S}) \subset \bigcup_{i=1}^{\infty} F\left(A \cap B_{r_{i}}\left(x_{i}\right)\right)
$$

By (4.23),

$$
\operatorname{diameter}\left(F\left(A \cap B_{r_{i}}\left(x_{i}\right)\right) \leq 2^{\alpha} C r_{i}^{\alpha},\right.
$$

and so

$$
\sum_{i=1}^{\infty}\left(\operatorname{diameter}\left(F\left(A \cap B_{r_{i}}\left(x_{i}\right)\right)\right)^{n-1} \leq 2^{(n-1) \alpha} C^{n-1} \sum_{i=1}^{\infty} r_{i}^{(n-1) \alpha} \leq 2^{(n-1) \alpha} C^{n-1} \gamma .\right.
$$

It follows that the Hausdorff $(n-1)$-measure of $\Gamma$ is less than a fixed multiple of $\gamma$ for every $\gamma$, which is a contradiction to Remark 4.3.

Suppose that $\|\cdot\|_{*}=\|\cdot\|_{p}$ where $1<p<\infty$. According Proposition 4.6, Remark A.1 and Theorem A.2, the distance formula holds if the Hausdorff $\theta$-measure of $\mathcal{S}$ is 0 where

$$
\begin{aligned}
& \text { (i) } \theta=(n-1) \frac{2}{p} \quad \text { if } \quad p \geq 2, \\
& \text { (ii) } \theta=(n-1) \frac{p}{2} \quad \text { if } \quad p \leq 2 .
\end{aligned}
$$

We compare these conditions to the requirement that $\mathcal{S}$ has 0 length. The requirement corresponding to (4.24) (i) is implied by 0 length provided that $2 \leq p$ and

$$
(n-1) \frac{2}{p} \geq 1 \quad \text { or } \quad 2(n-1) \geq p .
$$

The requirement corresponding to (4.24) (ii) is implied by 0 length provided that $1<p \leq 2$ and

$$
(n-1) \frac{p}{2} \geq 1 \quad \text { or } \quad p \geq \frac{2}{n-1} .
$$

Thus the range of $p$ for which "length 0 " suffices depends on $n$; if $n=2$ it is only $p=2$ while if $n \geq 3$, the range is $1<p \leq 2(n-1)$. In order to obtain the full range $1<p<\infty$, we formulate conditions which are satisfied by all the norms $\|\cdot\|_{p}, 1<p<\infty$, and under which we can prove the distance formula in the case that $\mathcal{S}$ has 0 length.

Definition 4.7. The norm $\|\cdot\|_{*}$ is geometrically convex if there is a $\Lambda>2$ such that

$$
\|x+2 y\|_{*}-\left\langle x+2 y, N^{*}(x)\right\rangle \geq \Lambda\left(\|x+y\|_{*}-\left\langle x+y, N^{*}(x)\right\rangle\right)
$$

for $\|x\|_{*}=1,\|y\|_{*} \leq 1$.

The norm $\|\cdot\|_{*}$ is balanced if there are constants $0<K, R$ such that

$$
\|x+y\|_{*}-\left\langle x+y, N^{*}(x)\right\rangle \leq K\left(\|x-y\|_{*}-\left\langle x-y, N^{*}(x)\right\rangle\right)
$$

for $\|x\|_{*}=1,\|y\|_{*} \leq R$. 
Remark 4.8. The notions "geometrically convex" and "balanced" were introduced in [2], wherein the restriction $\|y\|_{*} \leq 1$ following (4.27) is replaced by an apparently more general condition $\|y\|_{*} \leq r$ for some $0<r$ in the definition. However it is proved there that the more general condition is satisfied if and only if it is satisfied with $r=1$ and, perhaps, a different $\Lambda$. It is also proved in [2] that if $\|\cdot\|_{*}=\|\cdot\|_{p}, 1<p<\infty$, then $\|\cdot\|_{*}$ is geometrically convex and balanced. This does not seem to be obvious.

The geometical meaning of these conditions comes from the observation that

$$
\|x+y\|_{*}-\left\langle x+y, N^{*}(x)\right\rangle=\|x+y\|_{*}-\|x\|_{*}-\left\langle y, N^{*}(x)\right\rangle
$$

is the difference between $\|x+y\|_{*}$ and linear approximation of $\|\cdot\|_{*}$ at $x$ evaluated at $x+y$.

The following result is our deepest theorem, relying as it does on the deepest result of [2], adapted here for our current purpose in Theorem A.3.

Theorem 4.9. Let (4.27) and (4.28) hold. Let $\mathcal{S}$ have 0 length. Then the distance formula holds for all $x \in \mathcal{R}^{n}, r \in u\left(\mathcal{R}^{n}\right)$.

Proof. Via Lemma 3.1 and Remark 3.2, it suffices to treat regular values $r$ of $u$. Assuming that the distance formula does not hold, we rerun the Set Up. Since $\mathcal{S}$ has zero length, for every $\gamma>0$, we may cover $\mathcal{S}$ by countably many balls

$$
\mathcal{S} \subset \bigcup_{i=1}^{\infty} B_{\delta_{i}}\left(z_{i}\right)
$$

where $z_{i} \in \mathcal{S}$ and

$$
\sum_{i=1}^{\infty} \delta_{i} \leq \gamma
$$

Assume that the distance formula does not hold, and run the Set Up. We now freeze $i$ and attempt to estimate the area of $F\left(A \cap \bar{B}_{\delta_{i}}\left(z_{i}\right)\right)$. To this end, put $X=\Gamma$,

$$
Y=\{y:[y, x] \in A \text { for some } x \in \Gamma\},
$$

and $z_{0}=z_{i}, \delta=\delta_{i}$ in Theorem A.3. Then let $\mathcal{A}$ be given by (A.7). We next flesh out the other ingredients of Theorem A.3.

We may assume that $A \cap \bar{B}_{\delta_{i}}\left(z_{i}\right) \neq \emptyset$; equivalently, there exists $[y, x] \in \mathcal{A}$ such that $[y, x] \cap \bar{B}_{\delta_{i}}\left(z_{i}\right) \neq \emptyset$. According to the Set Up, as $z_{i} \in \mathcal{S}$, we then have

$$
\begin{aligned}
& \left\|z_{i}-x\right\|_{*} \geq\left\|z_{i}-x_{0}\right\|_{*}-\left\|x_{0}-x\right\|_{*} \geq 3 \epsilon-\epsilon=2 \epsilon, \\
& \left\|z_{i}-y\right\|_{*} \geq\left\|z_{i}-y_{0}\right\|_{*}-\left\|y_{0}-y\right\|_{*} \geq 3 \nu-\nu=2 \nu,
\end{aligned}
$$

where we used that $z_{i} \in \mathcal{S}$ and (4.9), (4.11). Therefore, if we take $\rho=\min (\epsilon, \nu)$, (A.6) is satisfied. Moreover, $3 \delta_{i}<\rho$ if $\gamma<\rho / 3$, as we now assume. Indeed, let us assume that $\gamma<\rho / 6$, and then

$$
\kappa=\frac{8}{\min (\epsilon, \nu)} \geq \frac{4}{\rho-3 \delta_{i}} .
$$


Theorem A.3 now yields the existence of $[\hat{x}, \hat{y}] \in \mathcal{A}$ which meets $\bar{B}_{\delta_{i}}\left(z_{i}\right)$ and $\hat{w}$ such that

$$
-\frac{\Lambda^{2} K}{\Lambda-2} \kappa \delta_{i} \leq\left\langle x-\bar{x}, N^{*}(\bar{w})\right\rangle \leq \frac{\Lambda^{2} K}{\Lambda-2} \kappa \delta_{i}
$$

whenever $[y, x] \in \mathcal{A},[y, x] \cap \bar{B}_{\delta_{i}}\left(z_{i}\right) \neq \emptyset$, and $\|x-\hat{x}\|_{*} \leq R$. This last condition is satisfied if $2 \epsilon<R$, which we may add to the conditions of the Set Up. Moreover, by (A.9),

$$
-\frac{\Lambda^{2} K}{\Lambda-2} \kappa \delta_{i} \leq\left\langle\hat{x}-\hat{y}, N^{*}(\hat{w})\right\rangle \leq 0 .
$$

From (4.34), the mapping $F$ of (4.17) satisfies

$$
F\left(A \cap \bar{B}_{\delta_{i}}\left(z_{i}\right)\right) \text { lies between parallel hyperplanes which are } C \delta_{i} \text { apart }
$$

where $C=2 \Lambda^{2} K \kappa /(\Lambda-2)$. Thus we expect the area of $F(A \cap \mathcal{S})$ to be estimable by $C^{\prime} \sum_{i=1}^{\infty} \delta_{i} \leq C^{\prime} \gamma$ for suitable $C^{\prime}$, and $\gamma$ can be made as small as desired. Since $\Gamma \subset F(A \cap \mathcal{S})$, this requires $\Gamma$ to have 0 area, which it does not, a contradiction.

However, there are more details to consider. In order that the area of the intersection of $\Gamma$ and a "strip" between parallel hyperplanes of width $2 C \delta$, say

$$
\Gamma \cap\left\{x:-C \delta \leq\left\langle x-\hat{x}, N^{*}(\hat{w})\right\rangle \leq C \delta\right\},
$$

be estimable in the form $C^{\prime} \delta$, we must quantify that the normal to the strip, here $N^{*}(\hat{w})$, does not approach the normal directions to $\Gamma$, and then provide the corresponding estimate. This is the role of (4.35). Thus we estimate the area of (4.37) subject to (4.35) (with $\delta_{i}=\delta$ ). From (4.35) and the Set Up, we infer

$$
\left\langle x_{0}-y_{0}, N^{*}(\hat{w})\right\rangle=\left\langle x_{0}-y_{0}-(\bar{x}-\bar{y}), N^{*}(\hat{w})\right\rangle+\left\langle\bar{x}-\bar{y}, N^{*}(\hat{w})\right\rangle
$$

and then

$$
\begin{aligned}
-C \delta-\epsilon-\nu \leq-C \delta-\|\left(x_{0}-\bar{x}\right) & -\left(y_{0}-\bar{y}\right) \|_{*} \leq\left\langle x_{0}-y_{0}, N^{*}(\hat{w})\right\rangle \\
& \leq\left\|\left(x_{0}-\bar{x}\right)-\left(y_{0}-\bar{y}\right)\right\|_{*} \leq \epsilon+\nu .
\end{aligned}
$$

We may assume that $\epsilon, \nu, \delta$ have been chosen so that this implies

$$
\left|\left\langle x_{0}-y_{0}, N^{*}(\hat{w})\right\rangle\right| \leq \frac{1}{2}\left|x_{0}-y_{0}\right|\left|N^{*}(w)\right| .
$$

Choose an orthogonal basis $e^{1}, \ldots, e^{n-1}$ of the space $Z$ of (4.6) for which

$$
e^{1} \text { is the orthogonal projection of } N^{*}(\hat{w}) \text { on } Z \text { and }\left|e^{j}\right|=1, j=2, \ldots, n-1 \text {. }
$$

Any $x \in \mathcal{R}^{n}$ can be uniquely written

$$
x=x_{0}+a_{0}\left(\left(x_{0}-y_{0}\right)-\frac{\left\langle x_{0}-y_{0}, N^{*}(\hat{w})\right\rangle}{\left\langle e^{1}, N^{*}(\hat{w})\right\rangle} e^{1}\right)+a_{1} N^{*}(\hat{w})+a_{2} e^{2}+\cdots+a_{n-1} e^{n-1} .
$$

Indeed, one easily calculates

$$
\begin{aligned}
& a_{0}=\frac{\left\langle x-x_{0}, x_{0}-y_{0}\right\rangle}{\left|x_{0}-y_{0}\right|^{2}}, a_{1}=\frac{\left\langle x-x_{0}, N^{*}(\hat{w})\right\rangle}{\left|N^{*}(\hat{w})\right|^{2}}, \\
& a_{j}=\left\langle x-x_{0}, e^{j}\right\rangle \text { for } j=2, \ldots, n-1 .
\end{aligned}
$$


Observe that if $x$ is in the set (4.37), then

$$
\frac{1}{\left|N^{*}(\hat{w})\right|^{2}}\left(-C \delta+\left\langle x_{0}-\bar{x}, N^{*}(\hat{w})\right\rangle\right) \leq a_{1} \leq \frac{1}{\left|N^{*}(\hat{w})\right|^{2}}\left(C \delta+\left\langle x_{0}-\bar{x}, N^{*}(\hat{w})\right\rangle\right) ;
$$

that is, $a_{1}$ is confined to an interval of width comparable to $\delta$.

If instead we use the orthogonal coordinates

$$
x-x_{0}=z_{0}\left(x_{0}-y_{0}\right)+z_{1} e^{1}+\ldots+z_{n-1} e^{n-1},
$$

where, here, now, the $z_{j}$ are numbers (and not vectors; in particular, they are not the centers of balls as above), then the $z^{\prime}$ s are given in terms of the $a^{\prime}$ s by

$$
z_{0}=a_{0}, z_{j}=a_{j} \text { for } j=2, \ldots, n-1,
$$

and, taking the inner-product (4.42) with $N^{*}(w)$ and using (4.40),

$$
\begin{aligned}
a_{1}\left|N^{*}(\hat{w})\right|^{2}= & \left\langle x-x_{0}, N^{*}(w)\right\rangle=z_{0}\left\langle x_{0}-y_{0}, N^{*}(\hat{w})\right\rangle \\
& +z_{1}\left\langle e^{1}, N^{*}(\hat{w})\right\rangle=a_{0}\left\langle x_{0}-y_{0}, N^{*}(\hat{w})\right\rangle+z_{1}\left\langle e^{1}, N^{*}(\hat{w})\right\rangle .
\end{aligned}
$$

That is

$$
z_{1}=\frac{a_{1}\left|N^{*}(\hat{w})\right|^{2}-a_{0}\left\langle x_{0}-y_{0}, N^{*}(\hat{w})\right\rangle}{\left\langle e^{1}, N^{*}(\hat{w})\right\rangle} .
$$

The condition that $x \in \Gamma$ entails

$$
z_{0}=g\left(z_{1}, \ldots, z_{n-1}\right)
$$

where $g$ is obtained from Proposition 4.2. This representation in terms of a graph becomes a parameterized representation in terms of the $a^{\prime}$ s:

$$
\left(a_{0}, a_{1}, \ldots, a_{n-1}\right) \mapsto\left(g\left(f\left(a_{0}, a_{1}\right), a_{2}, \ldots, a_{n-1}\right), f\left(a_{0}, a_{1}\right), a_{2}, \ldots, a_{n-1}\right) .
$$

where $f\left(a_{0}, a_{1}\right)$ is the right hand side of (4.44). The rigamarole around (4.38) was just to guarantee that the numerator $\left\langle e^{1}, N^{*}(\hat{w})\right\rangle$ is bounded away from 0 . In this regard, recall that $e^{1}$ was chosen to be the projection of $N^{*}(\hat{w})$ on the plane orthogonal to $x_{0}-y_{0}$. Everything in sight is Lipschitz, with controlled constants, and then the area formula for parameterized surfaces (e.g., Section 3.3 of Evans and Gariepy [8]) and the condition (4.41), which restricts $a_{1}$ to an interval of width comparable to $\delta$, now insure that the area of (4.37) is estimable in the form $C^{\prime} \delta$.

\section{Appendix A. Estimates from the Problem of Two Sticks}

In this appendix we present some consequences of developments in [2] in forms adapted to the current notation and uses.

We begin by considering norms $\|\cdot\|_{*}$ for which there are constants $0<A, B, q$, $p$, with $1<q \leq p$, such that

$$
A\|e-\bar{e}\|_{*}^{p} \leq 2-\|e+\bar{e}\|_{*} \quad \text { for } \quad\|e\|_{*}=\|\bar{e}\|_{*}=1
$$


that is, $\|\cdot\|_{*}$ is " $p$-uniformly convex," and

$$
\|x+y\|_{*}+\|x-y\|_{*}-2\|x\|_{*} \leq B\|y\|_{*}^{q} \quad \text { for } \quad\|x\|_{*}=1,
$$

that is, $\|\cdot\|_{*}$ is " $q$-uniformly smooth."

Remark A.1. If $2 \leq p<\infty$, the $p$-norm $\|\cdot\|_{p}$ is 2 -uniformly smooth and $p$-uniformly convex, while if $1<p \leq 2$, it is 2-uniformly convex, and $p$-uniformly smooth. The first assertion was proved by Clarkson [4] and the second by Hanner [10]. Regarding the more general and precise notions of "modulus of convexity" and "modulus of smoothness" and relations between them, see Lindenstrauss [12].

Theorem A.2. Let (A.1), (A.2) hold and $R>0$. Then there is a constant $C=C(A, B, R, p, q)$ such that if $y, x, \hat{y}, \hat{x}$, satisfy the conditions

$$
1=\|x-y\|_{*}=\|\hat{x}-\hat{y}\| \leq \min \left(\|x-\hat{y}\|_{*},\|\hat{x}-y\|_{*}\right)
$$

and

$$
\|x-\hat{x}\|_{*} \leq R,
$$

then

$$
\|x-\hat{x}\|_{*} \leq \frac{1}{t} C\left\|[y, x]_{t}-[\hat{y}, \hat{x}]_{s}\right\|_{*}^{q / p} \quad \text { for } \quad 0<t \leq s \leq 1 .
$$

The next result is less transparent. It follows immediately from results in [2], applied two sticks at a time. In the statement, we have in mind $Y=L_{r}, X=\Gamma$, as in the Set Up in the main text.

Theorem A.3. Let $Y, X \subset \mathcal{R}^{n}$ be nonempty and closed, $X$ be compact, and $d(x, Y)=1$ for $x \in X$. Let $\|\cdot\|_{*}$ be geometrically convex with parameters $1, \Lambda$, that is, let (4.27) hold, and balanced with constants $R, K$. Let $\rho>0, z_{0} \in \mathcal{R}^{n}$ and

$$
\rho<\min \left(d\left(z_{0}, X\right), d\left(z_{0}, Y\right)\right)
$$

Let

$$
\mathcal{A}=\left\{[y, x]: x \in X, y \in Y,\|x-y\|_{*}=1\right\}
$$

Let $0<3 \delta<\rho$ and

$$
\frac{4}{\rho-3 \delta} \leq \kappa
$$

Suppose that $[y, x] \cap \bar{B}_{\delta}\left(z_{0}\right) \neq \emptyset$ for some for some $[y, x] \in \mathcal{A}$. Then there exists $[\hat{y}, \hat{x}] \in \mathcal{A}$ such that $[\hat{y}, \hat{x}] \cap \bar{B}_{\delta}\left(z_{0}\right) \neq \emptyset$, and $\hat{w} \in \mathcal{R}^{n}$ with the properties below.

If $[y, x] \in \mathcal{A},[y, x] \cap \bar{B}_{\delta}\left(z_{0}\right) \neq \emptyset$, and $\|x-\hat{x}\|_{*} \leq R$, then

$$
-\frac{K \Lambda^{2}}{\Lambda-2} \kappa \delta \leq\left\langle x-\hat{x}, N^{*}(\hat{w})\right\rangle \leq \frac{K \Lambda^{2}}{\Lambda-2} \kappa \delta .
$$

Moreover,

$$
\|\hat{w}\|_{*}=\frac{K \Lambda^{2}}{\Lambda-2} \kappa \delta \quad \text { and } \quad-\frac{K \Lambda^{2}}{\Lambda-2} \kappa \delta \leq\left\langle\hat{x}-\hat{y}, N^{*}(\hat{w})\right\rangle \leq 0
$$


Remark A.4. For any $[y, x] \in \mathcal{A}$, the quantity

$$
\sigma(x-y, \kappa \delta):=\max \left\{\|x-y+w\|_{*}-1-\left\langle w, N^{*}(x-y)\right\rangle:\|w\|_{*} \leq \kappa \delta\right\}
$$

is defined. The "special" element $[\hat{y}, \hat{x}] \in \mathcal{A}$ in Theorem A.3 is determined by asking that $\sigma(\hat{x}-\hat{y}, \kappa \delta)$ be maximal among the elements of $\mathcal{A}$ which meet $\bar{B}_{\delta}\left(z_{0}\right)$; such elements exist owing to the obvious compactness of $\mathcal{A}$. Then $\hat{w}$ may be taken as any maximizing $w$ in the definition of $\sigma\left(\hat{x}-\hat{y}, K \Lambda^{2} \kappa \delta /(\Lambda-2)\right)$.

\section{REFERENCES}

[1] Aronsson, G. (1967). Extension of functions satisfying Lipschitz conditions. Ark. Mat. 6: 551-561.

[2] Caffarelli, L., Crandall, M. G. (preprint). The problem of two sticks.

[3] Cannarsa, P., Sinestrari, C. (2004). Semiconcave Functions, Hamilton-Jacobi Equations and Optimal Control, Progress in Nonlinear Differential Equations and Their Applications 58, Berlin:Birkhaäuser.

[4] Clarkson, J. A. (1936). Uniformly Convex Spaces, Trans. Amer. Math. Soc. 40:396-414.

[5] Clarke, F. H. (1976). On the inverse function theorem, Pacific J. Math. 64:97-102.

[6] Crandall, M. G. (2008). A visit with the $\infty$-Laplace equation, in Calculus of Variations and Non-Linear Partial Differential Equations, B. Dacorogna and P. Marcelli, eds, Lectures Notes in Mathematics 1927, Springer:Berlin.

[7] Crandall, M. G. and Evans, L. C. (2001). A remark on infinity harmonic functions, Electron. J. Differ. Equ. Conf. 6:123-129.

[8] Evans, Lawrence, Gariepy, Ronald F. (1992). Measure theory and fine properties of functions., Studies in Advanced Mathematics. CRC Press:Boca Raton.

[9] Fitzpatrick, S. (1984)). Differentiation of real-valued functions and continuity of metric projections. Proc. Amer. Math. Soc. 91:544-548.

[10] Hanner, O. (1956). On the uniform convexity of $L^{p}$ and $l^{p}$. Ark. Mat. 3:239-244.

[11] Khavinson, D. (1995). A note on entire solutions of the eiconal [eikonal] equation. Amer. Math. Monthly 102:159-161.

[12] J. Lindenstrauss (1963). On the modulus of smoothness and divergent series in Banach spaces, Michigan Math. J. 10:241252.

[13] Lions, P. L. (1982). Generalized Solutions of Hamilton-Jacobi Equations, Research Notes in Mathematics 69, Pitman:Boston.

Department of Mathematics, University of Texas at Austin, Austin, TX, 78712

E-mail address: caffarel@math.utexas.edu

Department of Mathematics, University of California, Santa Barbara, Santa Barbara, CA 93106

E-mail address: crandall@math.ucsb.edu 